\title{
Absorption and fluorescence spectra of poly(ethylene terephthalate) dimers
}

\author{
M. F. Sonnenschein and C. M. Roland \\ Chemistry Division, Code 6120, Naval Research Laboratory, Washington, \\ DC 20375-5000, USA
}

(Received 12 July 1989; revised 2 November 1989; accepted 20 November 1989)

\begin{abstract}
The concentration and temperature dependence of the absorption and fluorescence spectra of poly(ethylene terephthalate) (PET) solutions were measured. At concentrations above that associated with the onset of chain overlap, ground-state aggregation was observed. This aggregation phenomenon is similarly seen in the solid state, as well as in dimethyl terephthalate solutions. Elevated temperature effects dissociation of PET dimers, although their fluorescence intensity increases, presumably due to enhanced energy transfer.
\end{abstract}

(Keywords: poly(ethylene terephthalate); dimers; fluorescence)

\section{INTRODUCTION}

Recently it was reported that concentrated solutions and films of poly(ethylene terephthalate) (PET) exhibited fluorescence emission from two ground-state entities ${ }^{1-3}$. It was hypothesized that the two fluorescing structures were the aromatic unit of the phthalate residue (herein referred to as the (monomer') and a ground-state aggregation of the same residue (herein referred to as the 'dimer'). While Soxhlet extraction to remove smallmolecule impurities from the PET did not alter the fluorescence spectra ${ }^{3}$, the possibility that the emission results from impurities bound to the polymer chain remained. Another series of experiments ${ }^{4-6}$ under a different set of conditions, primarily at lower concentrations, have also been described.

In order to probe the origins of the PET fluorescence in more detail, a series of solution-phase experiments were conducted to test the ground-state aggregation hypothesis. The results of fluorescence and absorption spectroscopic measurements are reported herein.

\section{EXPERIMENTAL}

Amorphous PET (additive-free pellets; intrinsic viscosity $[\eta]=0.94 \mathrm{dl} \mathrm{g}^{-1}$ ) was kindly provided by Mr Craig Trask of Allied Signal Inc. (Petersburg, VA) and used without purification or further characterization. Dimethyl terephthalate (DMT) of better than $99 \%$ purity from the Aldrich Chemical Co. was recrystallized from boiling acetone prior to use. Solutions of DMT were filtered through Fisher Q2 filter paper prior to use. An absence of microcrystals in the filtered solutions was confirmed by inspection with a Zeiss D-7082 transmitting light microscope using crossed polarizers. Better than $99 \%$ pure 1,1,1,3,3,3-hexafluoro-2-propanol (HFIP) from Aldrich was used without purification. Ultra-violet/ visible absorption spectra, background-corrected at each temperature, were obtained using a Perkin-Elmer 3840 Lambda Array spectrophotometer (with purported linearity to $O D=3$ ) mated to a Perkin-Elmer 7500 microcomputer. Fluorescence spectra were taken on a Spex Fluorolog 212 spectrofluorimeter with a $450 \mathrm{~W}$ high-pressure xenon lamp. The solvent HFIP exhibited no absorption above $225 \mathrm{~nm}$, and no fluorescence emission over any of the excitation wavelengths utilized in this study. All fluorescence measurements were made observing front-face emission with slit widths constant at $0.35 \mathrm{~mm}$. After $30 \mathrm{~min}$ lamp warm-up, lamp jitter was less than $5 \%$ and low-frequency intensity fluctuations were negligible.

Temperature-dependent absorption measurements employed a Forma Scientific 2095 bath, circulating temperature-controlled water through the fluorescence cell holder. Cell temperature was determined by monitoring a thermometer placed in a cell containing water within the cell holder. At the desired temperature, a closed cell containing PET/HFIP solution was placed in the cell holder and allowed to equilibrate for $20 \mathrm{~min}$ before measurements were made. Longer equilibration times gave quantitatively equivalent results. All measurements were made in order of ascending temperature. Samples were not routinely deaerated, since even the most concentrated solutions at the lowest temperatures showed negligible oxygen quenching, as a consequence of the very short fluorescence lifetimes $\left(\sim 10^{-9} \mathrm{~s}\right)$ of both monomer and dimer entities ${ }^{3}$. All concentrations are reported herein based on the concentration of the PET repeat unit.

\section{RESULTS AND DISCUSSION}

Absorption spectra and fluorescence excitation and emission spectra of PET films have been reported previously ${ }^{8,9}$. We have essentially reproduced this previous work. The spectra of PET dissolved in HFIP are qualitatively similar to that of PET films, but several crucial differences exist. In Figure 1 is shown the absorption spectrum of PET in HFIP under relatively dilute conditions. Using the assignments of ref. 9, the $\mathrm{A}_{1}-\mathrm{A}_{1}^{\prime}$ state (with maximum absorption at wavelength $\lambda_{\max }=290 \mathrm{~nm}$ ) and the $A_{1}-B_{2}^{\prime}$ state (with $\lambda_{\max }$ at $244 \mathrm{~nm}$ ) exhibit red shifts of 10 and $14 \mathrm{~nm}$, respectively, compared with the film. The extinction coefficients are $\sim 10^{4}$ and $\sim 10^{5} \mathrm{~cm}^{-1} \mathrm{~mol}^{-1} 1$, respectively, in HFIP, in contrast to 
the nearly equal extinction coefficients measured for films ${ }^{9}$.

Previous workers have reported that observation of emission at $338 \mathrm{~nm}$ yielded a fluorescence excitation spectrum with $\lambda_{\max }$ at $321 \mathrm{~nm}$ (assigned to the monomer), while observation of emission at $390 \mathrm{~nm}$ obtained a fluorescence excitation spectrum with $\lambda_{\max }$ at $340 \mathrm{~nm}$ (assigned to the dimer) with measurable excitation out to $380 \mathrm{~nm}^{1,3}$. Figure 2 shows the absorption spectrum between 330 and $400 \mathrm{~nm}$ from a concentrated solution

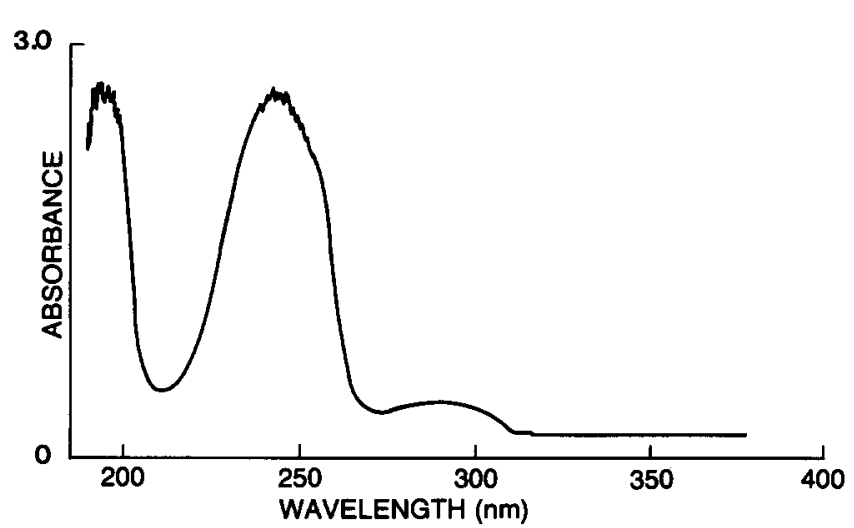

Figure 1 Room-temperature absorption spectrum of $5 \times 10^{-5} \mathrm{M}$ (by monomer) PET in HFIP. The absorption at wavelengths less than $220 \mathrm{~nm}$ is due to HFIP

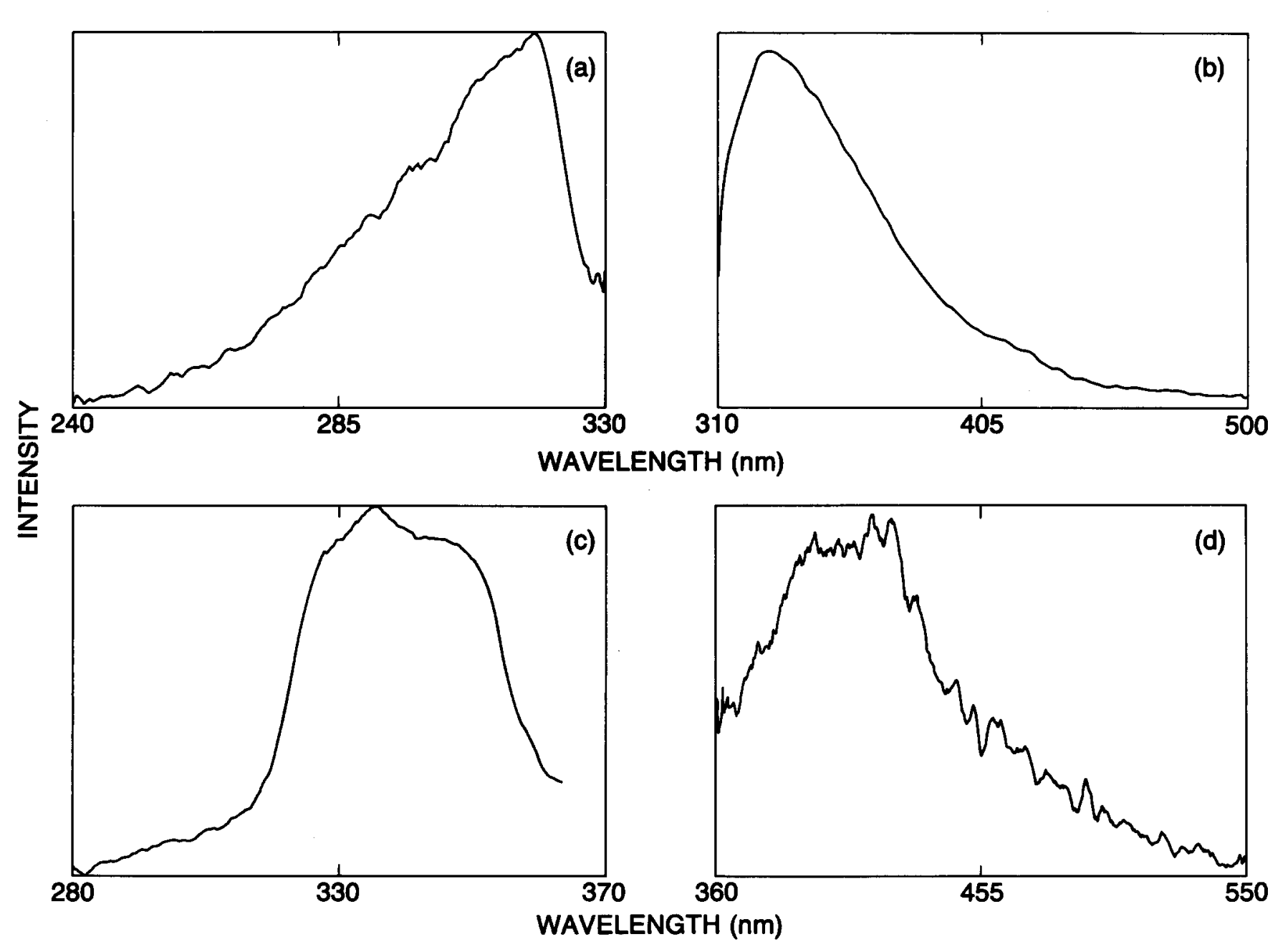

Figure 3 Excitation and emission spectra of $0.3 \mathrm{M}$ PET in HFIP: (a) monomer excitation, $\lambda_{\mathrm{em}}=340 \mathrm{~nm}$; (b) monomer emission, $\lambda_{\mathrm{ex}}=300 \mathrm{~nm}$; (c) dimer excitation, $\lambda_{\mathrm{em}}=390 \mathrm{~nm}$; (d) dimer emission, $\lambda_{\mathrm{ex}}=340 \mathrm{~nm}$ of PET in HFIP. The fluorescence excitation associated with emission at $390 \mathrm{~nm}$ is associated with the absorbing tail of the $A_{1}-A_{1}^{\prime}$ absorption. The resulting solutionphase fluorescence excitation and emission spectra associated with the absorbing electronic transitions and the tail absorption are presented in Figure 3.

To determine if the origin of the dimer excitation might actually be due to hot band absorption, the temperature dependence of the long-wavelength absorption was measured. An increase with solution temperature is, of

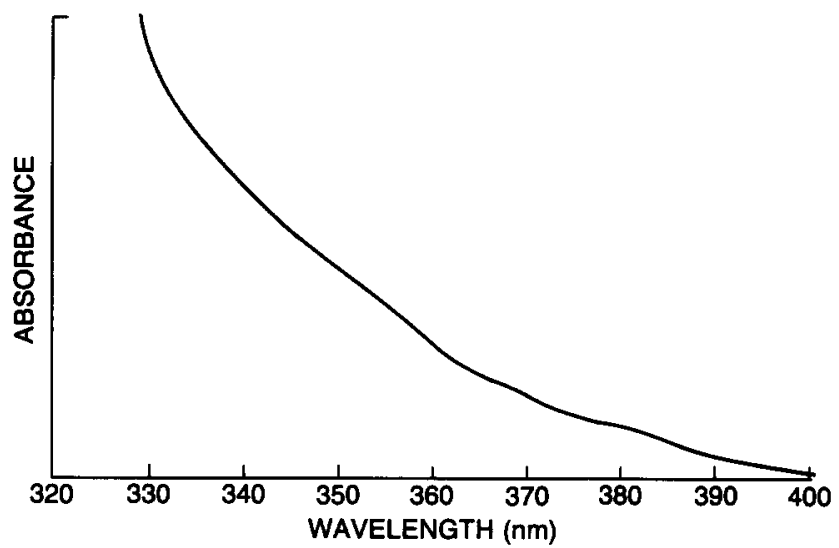

Figure 2 Absorption spectrum of $0.4 \mathrm{M}$ PET in HFIP at $32^{\circ} \mathrm{C}$. At wavelengths less than $320 \mathrm{~nm}$ the optical density is greater than 3 ; absorbance at $330 \mathrm{~nm}$ is 0.45 


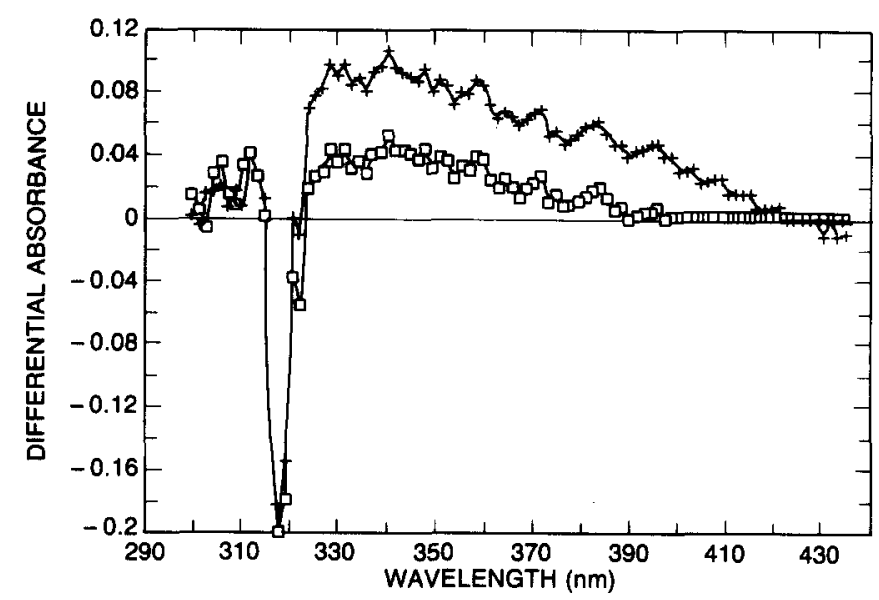

Figure 4 Differential absorption of 0.4 M PET in HFIP. Spectra are the difference in optical density at 24 and $32^{\circ} \mathrm{C}(\square)$ and at 24 and $45^{\circ} \mathrm{C}$ $(+)$. Absorption below $\sim 320 \mathrm{~nm}$ was beyond the linear range of the instrument

course, expected if the intensity represents a hot band absorption, while if the absorption is from ground-state dimers, their thermal dissociation at elevated temperature will result in an intensity reduction ${ }^{10}$. On the other hand, the absorption of impurities would be essentially unaffected by temperature*. Figure 4 shows the difference in absorption between that at 24 and $32^{\circ} \mathrm{C}$, and 24 and $45^{\circ} \mathrm{C}$. It is seen that, as the temperature is increased, the absorption of the tail decreases. A similar experiment, measuring the temperature dependence of the dimer fluorescence intensity, gave the opposite result; that is, dimer fluorescence increases with increasing temperature, consistent with previous results ${ }^{6,11}$. Since the reduction in dimer absorption with increasing temperature indicates thermal dissociation of the dimers, the increasing dimer fluorescence with temperature evidently results from thermally enhanced energy transfer ${ }^{3,12}$.

An experiment was performed to determine if the fluorescence intensity of the dimer emission was related in a predictable way to the total concentration of polymer. Because of energy transfer from the monomer excited state to the dimer excited state, it is not possible to derive relative concentrations from the fluorescence intensities; nevertheless, these measurements can provide insight into the nature of the aggregation process. Spectral intensity resulting from intrachain dimer formation would be consistent with a zero intercept for the emission intensity versus concentration, while interchain dimers would give rise to non-zero intercept. Figure 5 shows that the dimer fluorescence intensity does, in fact, extrapolate to a non-zero (and positive) concentration intercept. These data suggest that the origin of the dimer fluorescence is primarily aggregation between different polymer chains. Also, it is noted that the monomer fluorescence intensity has a strong negative correlation with this intensity, as expected if the latter is due to dimers rather than impurities.

Even in the melt, a polymer chain occupies a small portion of the volume it pervades ${ }^{13}$. In a good solvent, the segment density for a single chain is very low; hence,

* The volume expansion of HFIP over the temperature range is expected to be less than $3 \%$, assuming corresponding states with water between the melting and boiling points. The actual changes in the absorption are too large to be accounted for in this way interchain dimer formation is expected to dominate. The PET, with a weight-average molecular weight equal to 75000 (based on its intrinsic viscosity ${ }^{14}$ ), will have a radius of gyration $\left(R_{\mathrm{G}}\right)$ equal to $23 \mathrm{~nm}$ under theta conditions $^{15}$. This corresponds to a chain overlap concentration of roughly $0.01 \mathrm{M}$ in monomert. Figure 5 shows that appreciable dimer fluorescence intensities do, in fact, require concentrations beyond $0.01 \mathrm{M}$. Because of the strong concentration dependence of $R_{\mathrm{G}}$, the absolute transition from the dilute to semidilute concentration regime cannot be calculated; nevertheless, the congruence between the calculated and measured concentrations for chain overlap is instructive. Further results suggestive of a marked growth of PET dimer fluorescence at the dilute to semidilute concentration transition will be reported later ${ }^{16}$.

The dimer formation seen in PET should also occur in chemically similar small molecules. In fact, dimer fluorescence has been reported in fluorescence spectra taken of crystals of dimethyl terephthalate ${ }^{3}$. Although, previously, no evidence of aggregation was obtained from solutions of DMT in various solvents ${ }^{3}$, more concentrated solutions of DMT in either HFIP or chloroform do exhibit dimer fluorescence (Figure 6), as well as energy transfer from the monomeric lumophore to the groundstate aggregate. DMT can thus serve as a model compound for the crystal and solution photophysics of PET.

\section{SUMMARY}

Experimental observations have been presented that are consistent with the hypothesis that the long-wavelength excitation/emission spectra of PET are associated primarily with interchain ground-state aggregation. Under the conditions reported in this paper, the ground-state absorption and its inverse dependence on temperature, when coupled with the reported absence of a 'growing-in' of dimer fluorescence ${ }^{3,17}$, provide strong evidence for the presence of PET ground-state aggregates in solution. The increase in the long-wavelength fluorescence intensity with increasing temperature, notwithstanding thermal

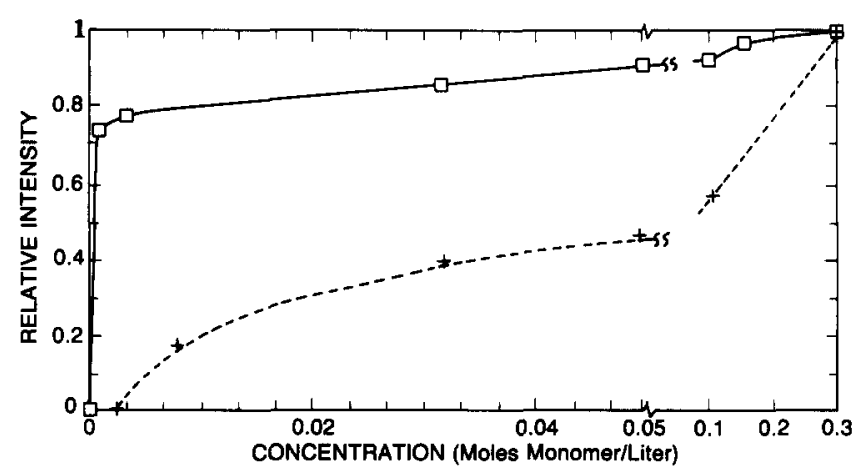

Figure 5 Concentration dependence of monomer (-) and dimer $(---)$ integrated emission intensities. Values in each series are normalized to the solution of highest fluorescence intensity. Monomer spectra are corrected for the slight change in fluorescence excitation spectrum as a function of concentration in this range. See footnote to text for details

$\dagger c^{*}$ is calculated using $R_{\mathrm{G}}$ as the radius of a sphere that the polymer in solution excludes. There is no a priori reason to choose any particular model for the calculation of $c^{*}$ in these experiments, but the method chosen here reflects in part their chain dynamic aspect 

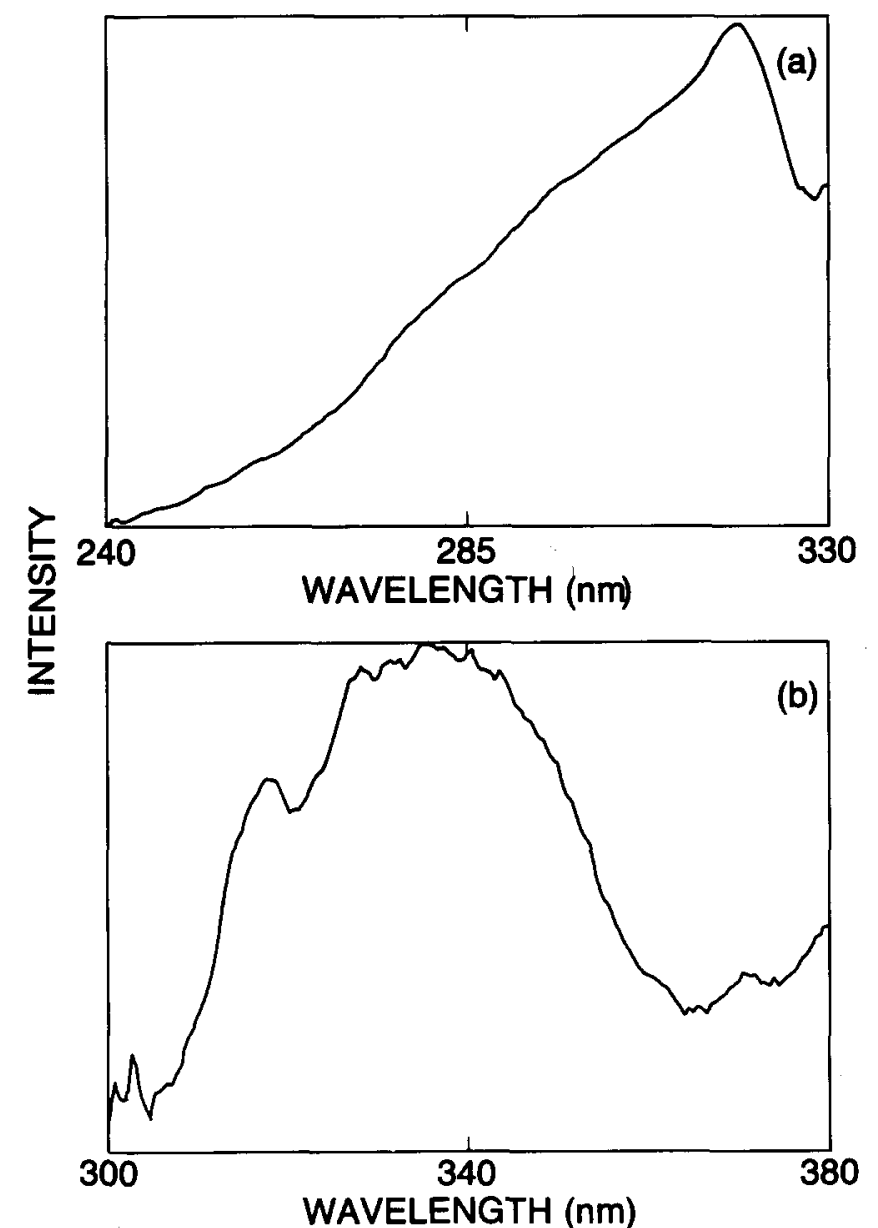

Figure 6 Excitation spectra of DMT-saturated HFIP: (a) monomer spectrum, $\lambda_{\mathrm{em}}=340 \mathrm{~nm}$; (b) dimer, $\lambda_{\mathrm{em}}=450 \mathrm{~nm}$. Energy transfer from the monomer to the dimer is evidenced by the presence of the monomer spectrum $(<330 \mathrm{~nm})$ in the dimer excitation. Emission spectra are substantially similar to those in Figure 3, but reflect a greater overlap of the absorption spectra than in PET

dissociation of the dimers, probably reflects an enhancement in the energy transfer from monomers to dimers. The growth of monomer fluorescence intensity is negatively correlated with that of the dimer, while the positive, non-zero intercept of the fluorescence intensity when plotted as a function of polymer concentration suggests primarily interchain aggregation. The implications of this ground-state aggregation on the rheological behaviour of PET in solution and in the melt remain to be investigated.

\section{ACKNOWLEDGEMENTS}

M.S. thanks the National Research Council for a post-doctoral fellowship.

\section{REFERENCES}

1 Allen, N. S. and McKeller, J. F. Makromol. Chem. 1978, 179, 523

2 Hennecke, M. and Fuhrmann, J. Makromol. Chem., Macromol. Symp. 1986, 5, 181

3 Hemker, D. J., Frank, C. W. and Thomas, J. W. Polymer 1988, 29, 437

4 Takai, Y., Misutani, T. and Ieda, M. Japan. J. Appl. Phys. 1978, 17, 651

5 Renyuan, Q. in 'Macromolecules" (Eds. H. Benoit and P. Rempp), Pergamon Press, Oxford, 1982

6 Mendicuti, F., Patel, B., Vellarkad, V. N. and Mattice, W. L. Polymer 1989, 29, 1669

7 Hirayama, F. J. Chem. Phys. $1965,42,3163$

8 Phillips, D. J. and Schug, J. C. J. Chem. Phys. 1969, 50, 3297

9 Padhye, M. R. and Tamhane, P. S. Angew. Makromol. Chem. $1978,69,33$

10 Irie, M., Kamijo, T., Alkawa, M., Takemura, T., Hayashi, K. and Baba, H. J. Phys. Chem. 1977, 81, 1571

11 Hennecke, M., Kurz, K. and Fuhrmann, J. Polymer 1989, 30, ???

12 MacCallum, J. R. in 'Photophysics of Polymers' (Eds. C. E. Hoyle and J. M. Torkelson), American Chemical Society, Washington DC, 1987

13 deGennes, P. G. 'Scaling Concepts in Polymer Physics', Cornell University Press, Ithaca, NY, 1979

14 Weisskopf, K.J.Polym. Sci., Polym. Chem. Edn. 1988, 26, 1919

15 Naoki, M., Park, I.-H., Wunder, S. L. and Chu, B. J. Polym. Sci., Polym. Phys. Edn. 1985, 23, 2567

16 Sonnenschein, M. F. and Roland, C. M. J. Polym. Sci., Polym. Phys. Edn. submitted for publication

17 Birks, J. B. 'Photophysics of Aromatic Molecules', Wiley, New York, 1970 